\title{
The utility of ezetimibe therapy in nonalcoholic fatty liver disease
}

Baek Gyu Jun and Gab Jin Cheon

Department of Internal Medicine, Gangneung Asan Hospital, University of Ulsan College of Medicine, Gangneung, Korea

Received: January 30, 2019

Accepted: February 18, 2019

\section{Correspondence to}

Gab Jin Cheon, M.D.

Division of Gastroenterology, Department of Internal Medicine, Gangneung Asan Hospital, University of Ulsan College of Medicine, 38 Bangdong-gil, Gangneung 25440, Korea

Tel: $+82-33-610-3100$

Fax: +82-33-644-5495

E-mail:1000@gnah.co.kr

\section{See Article on Page 296-304}

Nonalcoholic fatty liver disease (NAFLD), which is defined as the presence of more than $5 \%$ hepatic steatosis, is an emerging liver disease in developed countries [1]. NAFLD is classified as nonalcoholic fatty liver (NAFL), which is not associated with inflammation, or nonalcoholic steatohepatitis (NASH), which is associated with inflammation and fibrosis. NAFL is a benign liver disease with a good prognosis, whereas NASH can progress to liver fibrosis, cirrhosis, and hepatocellular carcinoma [2]. Therefore, many clinical trials of therapies for NAFLD have been conducted. However, there has been no established therapy for NAFLD until recently.

NAFLD is strongly associated with obesity, diabetes mellitus, and dyslipidemia [3]. Therefore, it is important to treat dyslipidemia and control glucose levels in patients with NAFLD. Ezetimibe is a lipid-lowering drug that inhibits the absorption of intestinal cholesterol by binding to Niemann-Pick C1-like 1, a cholesterol transporter [4]. In a previous study, ezetimibe ameliorated hepatic steatosis and dyslipidemia in an animal model [5]. Therefore, it is anticipated that ezetimibe treatment will have positive effects in patients with NAFLD.
In a meta-analysis within the current issue of Korean Journal of Internal Medicine, Lee et al. [6] analyzed patients with biopsy-proven NAFLD and reported that ezetimibe decreased the NAFLD activity score (NAS) but did not improve hepatic steatosis. An improvement in the NAS suggests a decrease in NASH-associated inflammation, and thereby prevention of cirrhosis. That meta-analysis also demonstrated the safety of ezetimibe in terms of side effects, and ezetimibe did not induce an increase in glucose or glycated hemoglobin ( $\mathrm{HbArc}$ ) levels. Ezetimibe seemed useful for treating NAFLD with dyslipidemia.

In the first randomized controlled clinical trial of ezetimibe for NAFLD, Takeshita et al. [7] reported that ezetimibe improved liver pathology; however, that study was limited by a small sample size ( 32 patients). In a meta-analysis, Nakade et al. [8] showed that ezetimibe improved liver enzyme levels, the severity of hepatic steatosis, and ballooning. In a study assessing the impact of ezetimibe on cardiovascular morbidity and mortality, ezetimibe/simvastatin use was associated with less elevation of liver enzyme levels $(p=0.037)$ and reduced gamma-glutamyl transpeptidase levels $(p=0.014)$ compared with placebo/simvastatin [9]. That study showed that combination 
treatment with ezetimibe and a statin is safe and effective for improving liver enzyme levels. Taken together, these studies suggest that ezetimibe treatment has benefits in normalizing liver enzyme levels and improving hepatic steatosis.

However, ezetimibe has not shown positive results in all previous studies. In a randomized trial that assessed changes in liver fat using magnetic resonance imaging and elastography, ezetimibe did not significantly improve hepatic steatosis in NASH or liver histology [10]. A meta-analysis of six open-label single-arm randomized controlled trials indicated improvements in liver enzyme levels but not in hepatic inflammation or fibrosis [8]. Therefore, ezetimibe treatment for patients with NAFLD remains controversial due to these conflicting results. Moreover, most of the studies that showed improvements in NAFLD by ezetimibe treatment were performed in a preclinical setting [5,11].

The side effects of ezetimibe on glucose metabolism should always be taken into consideration when treating patients with NAFLD. A previous study showed that ezetimibe influenced glucose metabolism in an animal model [5]. Ezetimibe treatment poses a risk of new-onset diabetes and increased HbArc levels [12]. However, a recent meta-analysis reported that ezetimibe does not affect glucose or $\mathrm{HbA1c}$ levels, demonstrating the safety of ezetimibe treatment for patients with NAFLD [13].

Ezetimibe therapy is expected to have an effect on NAFLD/NASH according to previous studies, including the meta-analysis by Lee et al. [6] However, ezetimibe treatment for patients with NAFLD/NASH remains controversial because most previous clinical trials conducted were single arm or had small sample sizes. Furthermore, most previous meta-analyses analyzed few randomized controlled trials. Therefore, a large-scale randomized controlled study is needed to confirm the effects of ezetimibe on NAFLD.

\section{Conflict of interest}

No potential conflict of interest relevant to this article was reported.

\section{REFERENCES}

1. Loomba R, Sanyal AJ. The global NAFLD epidemic. Nat Rev Gastroenterol Hepatol 2013;10:686-690.

2. Pais R, Charlotte F, Fedchuk L, et al. A systematic review of follow-up biopsies reveals disease progression in patients with non-alcoholic fatty liver. J Hepatol 2013;59:550-556.

3. Souza MR, Diniz Mde F, Medeiros-Filho JE, Araujo MS. Metabolic syndrome and risk factors for non-alcoholic fatty liver disease. Arq Gastroenterol 2012;49:89-96.

4. Filippatos TD, Mikhailidis DP. Lipid-lowering drugs acting at the level of the gastrointestinal tract. Curr Pharm Des 2009;15:490-516.

5. Muraoka T, Aoki K, Iwasaki T, et al. Ezetimibe decreases SREBP-1c expression in liver and reverses hepatic insulin resistance in mice fed a high-fat diet. Metabolism 2011;60:617-628.

6. Lee HY, Jun DW, Kim HJ, et al. Ezetimibe decreased nonalcoholic fatty liver disease activity score but not hepatic steatosis. Korean J Intern Med 2019;34:296-304.

7. Takeshita Y, Takamura T, Honda M, et al. The effects of ezetimibe on non-alcoholic fatty liver disease and glucose metabolism: a randomised controlled trial. Diabetologia 2014;57:878-890.

8. Nakade Y, Murotani K, Inoue T, et al. Ezetimibe for the treatment of non-alcoholic fatty liver disease: a meta-analysis. Hepatol Res 2017;47:1417-1428.

9. Simon TG, Corey KE, Cannon CP, et al. The nonalcoholic fatty liver disease (NAFLD) fibrosis score, cardiovascular risk stratification and a strategy for secondary prevention with ezetimibe. Int J Cardiol 2018;270:245-252.

10. Loomba R, Sirlin CB, Ang B, et al. Ezetimibe for the treatment of nonalcoholic steatohepatitis: assessment by novel magnetic resonance imaging and magnetic resonance elastography in a randomized trial (MOZART trial). Hepatology 2015;61:1239-1250.

11. Kim SH, Kim G, Han DH, et al. Ezetimibe ameliorates steatohepatitis via AMP activated protein kinase-TFEB-mediated activation of autophagy and NLRP3 inflammasome inhibition. Autophagy 2017;13:1767-1781.

12. Erqou S, Lee CC, Adler AI. Statins and glycaemic control in individuals with diabetes: a systematic review and meta-analysis. Diabetologia 2014;57:2444-2452.

13. Wu H, Shang H, Wu J. Effect of ezetimibe on glycemic control: a systematic review and meta-analysis of randomized controlled trials. Endocrine 2018;60:229-239. 